\title{
COMPATIBILIDAD DE Beauveria bassiana Y Metarhizium anisopliae CON Chrysoperla externa DEPREDADOR DE Trialeurodes vaporariorum
}

\section{COMPATIBILITY OF Beauveria bassiana AND Metarhizium anisopliae WITH Chrysoperla externa DEPREDATOR OF Trialeurodes vaporariorum}

\author{
Mayerly Alejandra Castro López ${ }^{*}$, John Wilson Martínez Osorio ${ }^{1}$ \\ ${ }^{1}$ Universidad Pedagógica y Tecnológica de Colombia (UPTC), Facultad de Ciencias Agrarias, Escuela \\ de Ingeniería Agronómica, Grupo de Investigación Manejo Biológico de Cultivos (GMBC), Av. Central \\ del Norte 39-115, Tunja-Boyacá, Colombia. \\ Autor de correspondencia E-mail: macastrol@unal.edu.co.
}

\section{RESUMEN}

Trialeurodes vaporariorum (Westwood) es una plaga clave en el cultivo de tomate (Solanum lycopersicum L.) que es controlada principalmente con insecticidas. En la actualidad existen en el mercado productos para su control biológico, como entomopatógenos y depredadores, pero no se utilizan de forma generalizada, requiriéndose más estudios que orienten su implementación. De acuerdo con esto, en el presente trabajo se evaluó la compatibilidad en condiciones de laboratorio de los hongos Beauveria bassiana (Balsamo) Vuill y Metarhizium anisopliae (Metsch) con Chrysoperla externa Hagen, depredador de ninfas de $T$. vaporariorum. Se empleó un diseño completamente al azar con ocho repeticiones y tres tratamientos, consistentes en concentraciones de $1 \times 10^{5}, 1 \times 10^{7}$ y $1 \times 10^{8}$ conidias $\mathrm{mL}^{-1}$ para cada entomopatógeno y un tratamiento testigo con agua destilada y Tween 80 $(0,1 \%)$ para cada uno de los estadios larvales I, II y III del depredador. Los resultados indicaron que no hubo diferencias estadísticas en la mortalidad corregida en los estadios larvales II y III de $C$. externa, expuestos a las diferentes concentraciones de B. bassiana y para el estado larval de $C$. externa a diferentes concentraciones de $M$. anisopliae $(P<0,05)$ evaluadas. La mortalidad estuvo por debajo del $15 \%$ para B. bassiana y del $10 \%$ para $M$. anisopliae. Estos resultados indican que C. externa podría ser empleada como entomófago en un esquema de manejo integrado de T. vaporariorum en cultivos de tomate, conjuntamente con hongos como B. bassiana y M. anisopliae.

Palabras clave: entomófago, hongos entomopatógenos, control biológico.

\section{ABSTRACT}

Trialeurodes vaporariorum (Westwood) is a key pest in tomato (Solanum lycopersicum L.) crops, and it is mainly controlled by insecticides. Nowadays, there are biological control products for this pest, such as predators and entomopathogenic fungi. However, they are not used extensively because more studies are required for their implementation. Therefore, this study evaluated the compatibility under laboratory conditions of Beauveria bassiana and Metarhizium anisopliae with Chrysoperla externa Hagen, a predator of $T$. vaporariorum nymphaea. The experiment was conducted using a completely randomized experimental design with three treatments and eight replicates. The treatments consisted of concentrations of $1 \times 10^{5}, 1 \times 10^{7}$ and $1 \times 10^{8}$ conidias $\mathrm{mL}^{-1}$ for each entomopathogen, and one control treatment with distillated water and Tween $80(0.1 \%)$ for larval stages I, II and III of the predator. No statistical differences were found in the corrected mortality of stages II and III of C. externa larvae exposed to the different concentrations of B. bassiana, or for the 
larval stage of $C$. externa exposed to different concentrations of $M$. anisopliae $(P<0.05)$. The mean mortality rates observed in the whole test were under $15 \%$ and $10 \%$ for B. bassiana and M. anisopliae, respectively. These results showed that $C$. externa could be used as an entomophagous insect into a $T$. vaporariorum integrated management system, combined with B. bassiana and M. anisopliae.

Key words: entomophagous, entomopathogenic fungi, biological control.

\section{INTRODUCCIÓN}

En el ámbito mundial una de las hortalizas más cultivadas es el tomate (Solanum lycopersicum L.) (Bai y Lindhout, 2007). En Colombia se sembraron 12.920 ha de esta hortaliza el año 2017 (Agronet, 2018), como resultado de la tecnificación del cultivo y la mejora en su comercialización hacia mercados nacionales e internacionales (Perrilla et al., 2011; Herrera et al., 2015)

Trialeurodes vaporariorum (Westwood) (Hemiptera: Aleyrodidae), o mosca blanca de los invernaderos, es una de las principales limitantes del cultivo de tomate (Barrera et al., 2013), generando importantes pérdidas económicas debido a que ocasiona daños directos e indirectos a las plantas (Martínez et al., 2012). La situación se hace más compleja porque algunas poblaciones de $T$. vaporariorum han adquirido resistencia a insecticidas piretroides, organofosforados y carbamatos, que son ampliamente utilizados para el control de este insecto plaga (Cardona et al., 2001; Jaramillo et al., 2007). Por lo expuesto anteriormente, el control biológico de este insecto se visualiza como una de las alternativas de manejo más adecuadas, porque permitiría minimizar el impacto sobre los organismos presentes en estos agroecosistemas, y producir alimentos no contaminados.

Algunas de las herramientas más usadas en el control biológico para reducir las poblaciones de artrópodos plaga son los insectos (depredadores y parasitoides) y microorganismos entomopatógenos (bacterias, virus, hongos y nemátodos), pero para que estos enemigos naturales puedan ser considerados como una alternativa viable dentro de un programa de manejo integrado para mosca blanca se hace necesario establecer si son compatibles entre sí (Estévez et al., 2011).

Dentro de los insectos depredadores empleados en control biológico se destacan las crisopas (Neuroptera: Chrysopidae), cuyas larvas se alimentan succionando la hemolinfa de artrópodos de tegumento fácil de perforar (Souza et al., 2015). Chrysoperla externa Hagen (1861) es una de las especies de mayor importancia, porque sus larvas presentan altas tasas de depredación, gran capacidad de búsqueda de la presa $y$, además, una amplia distribución neotropical (Gamboa et al., 2016).
Por otro lado, los hongos entomopatógenos Beauveria bassiana (Bals.) Vuill. y Metarhizium anisopliae (Metsch.) han sido reconocidos como importantes agentes de control para diversas plagas agrícolas, debido a su eficacia y facilidad de multiplicación en laboratorio (Téllez et al., 2009); el mecanismo de acción de estos entomopatógenos se basa en la acción de las conidias del hongo que entran en contacto con la exocutícula del insecto, forman un apresorio o estructura de adhesión, posteriormente crece el tubo germinativo que penetra por acción mecánica y rápidamente llega al hemocele, donde produce toxinas y enzimas, que provocan daños en los órganos internos del huésped, hasta ocasionar su muerte (Hajek, 1997; Monzón, 2001).

En estudios previos (Castro et al., 2016), se evaluó la capacidad de depredación de C. externa sobre ninfas de $T$. vaporariorum en condiciones de laboratorio, obteniendo porcentajes de mortalidad de $97 \%$, 94\% y $99 \%$ para el I, II y III estadio larval, respectivamente. Del mismo modo, $B$. bassiana y $M$. anisopliae se han mencionado como agentes importantes de control biológico para plagas de la familia Aleyrodidae, en cultivos de tomate en invernadero y campo, afectando principalmente estados ninfales. En Colombia se reportan porcentajes de mortalidad del $96 \%$ (Espinel et al., 2008) con B. bassiana; Schlick-Souza et al. (2011a), indicaron que M. anisopliae presentó una eficiencia de control del 53\% para mosca blanca en Brasil.

Se evidencia de esta manera que C. externa, $B$. bassiana y $M$. anisopliae constituyen organismos útiles en el control biológico y el manejo integrado de $T$. vaporariorum en el cultivo de tomate.

En Colombia pocos estudios han evaluado los efectos que se presentan con la implementación de insectos depredadores en asociación con hongos entomopatógenos en programas de manejo integrado de plagas (Amorim et al., 2005) y aún no es clara la posible interacción o interferencia que se pueda presentar entre los diferentes agentes de control biológico. Maia et al. (2000) mencionaron que cuando los hongos entomopatógenos entran en contacto con depredadores, se pueden presentar alteraciones como inviabilidad de huevos, alteración del ciclo de vida y disminución de la capacidad de depredación; esto ocurre porque los depredadores pueden ser, al igual que la plaga, afectados directamente por los hongos 
entomopatógenos. Hamdi et al. (2011) consideran que, a pesar de los estudios realizados aún falta información de laboratorio y campo sobre la susceptibilidad de depredadores hacia hongos entomopatógenos usados en programas de control biológico de plagas.

Por lo anterior, en función de evaluar la viabilidad del uso de depredadores asociados con hongos entomopatógenos, que permita manejar poblaciones de mosca blanca en cultivos de tomate, el presente trabajo tuvo como objetivo determinar en condiciones de laboratorio el posible efecto letal de los hongos B. bassiana y $M$. anisopliae sobre los tres estadios de C. externa.

\section{MATERIALES Y MÉTODOS}

La investigación se realizó en el laboratorio de Entomología del Grupo Manejo Biológico de Cultivos (GMBC), de la Universidad Pedagógica y Tecnológica de Colombia (UPTC), Tunja, Boyacá $\left(5^{\circ} 33^{\prime} 16^{\prime \prime} \mathrm{N}, 73^{\circ} 21^{\prime} 09^{\prime \prime} \mathrm{O}\right)$, Colombia, durante el 2016 , con una temperatura promedio de $20,1^{\circ} \mathrm{C}$ y humedad relativa del $55 \%$. Adicionalmente, se sembraron en condiciones de invernadero plantas de tomate Solanum lycopersicum L., las cuales se infestaron con individuos adultos de T. vaporariorum para emplear este material como fuente de alimentación para el depredador, para obtener cada uno de los estadios larvales durante las pruebas en laboratorio.

Los hongos entomopatógenos fueron obtenidos de dos productos comercializados en Colombia con registro otorgado por el Instituto Colombiano Agropecuario (ICA) entidad encargada de reglamentar y controlar la calidad de los insumos agrícolas, cuyo ingrediente activo para $B$. bassiana son 200 millones de conidias viables por $\mathrm{cm}^{3}$, con un recuento viable de $1,6 \times 10^{8}$ conidias por $\mathrm{cm}^{3}, 80 \%$ de viabilidad de conidias y una pureza microbiológica del $95 \%$; el producto cuyo ingrediente activo fue $M$. anisopliae presentó las mismas características; La presentación de $B$. bassiana era una suspensión concentrada (SC) y la de $M$. anisopliae era polvos mojables (WP).

Para corroborar la presencia de los hongos en los bioformulados se realizo un control de calidad a cada ingrediente activo, que consistió en siembras directas de B. bassiana y M. anisopliae en cajas de Petri que contenían medio Agar Papa Dextrosa (PDA), al medio de cultivo se le aplicó ácido láctico $(1 \%)$ suplementado con antibiótico (cloranfenicol) para evitar la contaminación por bacterias, a una temperatura de $23^{\circ} \mathrm{C}$ (Lucero et al., 2004), transcurridos ocho días se procedió a realizar la identificación morfológica mediante el trabajo de Barnett y Hunter. (1998). En el caso del depredador C. externa, éste se obtuvo de la empresa productora y comercializadora de productos de origen biológicos llamada Perkins Ltda., ubicado en la ciudad de Palmira, Colombia.

\section{Metodología de los bioensayos}

La determinación del efecto de los hongos se realizó en cajas de Petri cubiertas con velo suave, liviano y transparente, con cinco individuos por placa del estado larval del depredador C. externa a evaluar. La aplicación de cada hongo se realizó en cámara de flujo laminar, utilizando un atomizador calibrado con papel hidrosensible para obtener un cubrimiento homogéneo $\left(60\right.$ gotas $\left.\mathrm{cm}^{-2}\right)$. Se realizaron dos pases del aplicador sobre cada caja de Petri a una altura de $20 \mathrm{~cm}$, directamente sobre los individuos a tratar.

Se utilizaron suspensiones de los hongos, individualmente preparadas con agua destilada y Tween $80(0,1 \%)$ para favorecer la dispersión de las conidias; de cada suspensión se realizaron diluciones sucesivamente hasta $1 \times 10^{-3}$ de las cuales se tomaron $10 \mu \mathrm{L}$ para realizar los conteos en cámara Neubauer, con ayuda de un microscopio (Vélez et al., 1997) Nikon ${ }^{\circledR}$. La densidad fue ajustada para las concentraciones de $1 \times 10^{5}, 1 \times 10^{7} \mathrm{y}$ $1 \times 10^{8}$ conidias $\mathrm{mL}^{-1}$ para cada uno de los estadios larvales de C. externa, siguiendo la metodología descrita por Schlick-Souza et al. (2011b) y Estevez et al. (2011).

Por otro lado, se prepararon frascos de vidrio de $5 \mathrm{~mL}$ de capacidad con agar-agar $\left(13 \mathrm{~g} \mathrm{~L}^{-1}\right)$, el cual sirvió como medio de soporte e hidratación del material vegetal (foliolos de tomate del invernadero) empleado en las pruebas biológicas. Cada foliolo con su soporte se depositó en un recipiente plástico de $19 \mathrm{~cm}$ de ancho, $6 \mathrm{~cm}$ de alto, con una capacidad de $0,907 \mathrm{~kg}$, con cierre hermético.

Transcurrida una hora después de la aplicación de los hongos, los individuos de C. externa se trasladaron a los recipientes plásticos que contenían los foliolos de tomate con huevos $y$ ninfas de $T$. vaporariorum como fuente de alimentación; se depositó un individuo del estado larval de C. externa a evaluar (estadio larval I, II o III) por cada caja, lo anterior correspondió a la unidad experimental del ensayo.

A partir de 24 horas después de la aplicación de las esporas, se procedió a registrar diariamente el número de depredadores muertos, durante el tiempo de duración de cada estadio larval, que correspondió a 96 horas para el estadio larvar I y II, y de 192 horas para el estadio III.

Se realizó ajuste a los datos obtenidos, calculando el porcentaje de mortalidad corregida según la fórmula de Abbott (1925) así:

$$
\% \text { Mortalidad corregida }=\left(\frac{\% \mathrm{mt}-\% \mathrm{mta}}{100-\mathrm{mta}}\right) * 100
$$


dónde: $\% \mathrm{mt}=$ Porcentaje de mortalidad en el tratamiento; \%mta = Porcentaje de mortalidad en el testigo absoluto.

La misma metodología se empleó para la evaluación de B. bassiana y M. anisopliae.

La verificación del efecto de los hongos se realizó retirando los individuos muertos, los cuales se ubicaron en cámara húmeda, a una temperatura aproximada de $20^{\circ} \mathrm{C}$ y se hizo seguimiento para observar si crecía micelio sobre las larvas de C. externa. Posteriormente, a partir del micelio que aparecía desde los insectos muertos se realizaron siembras sobre medio PDA en cámara de flujo laminar, durante diez días. Lo anterior, con el fin de realizar la identificación morfológica y corroborar la presencia de los hongos entomopatógenos como agentes causantes de la mortalidad de los insectos (Bautista et al., 2014).

Los experimentos se desarrollaron con un diseño completamente al azar (DCA), con cuatro tratamientos (tres concentraciones y el testigo) y ocho repeticiones, para un total de 32 unidades experimentales por entomopatógeno y estadio, las pruebas se realizaron independientemente para cada ingrediente activo y para cada estadio de C. externa. Los datos se analizaron con el programa SAS v. 9.2 (SAS Institute Inc., Cary, North Caroline, USA). Se realizó Análisis de Varianza (ANOVA) para detectar diferencias entre tratamientos, verificando previamente los supuestos de homogeneidad de varianzas y normalidad mediante las pruebas deShapiro-Wilk y Levene, respectivamente. En la comparación de medias se utilizó la prueba de Tukey, con un nivel se significancia de $\alpha=0,05$.

Para determinar el impacto de B. bassiana y $M$. anisopliae sobre el estado larval de C. externa se empleó la escala de toxicidad a enemigos naturales aplicada por la Organización Internacional para el Control Biológico e Integrado de los Animales y Plantas perjudiciales para los cultivos (OILB) (IOBC/WPRS, 1992; Viñuela et al., 1993), como se observa a continuación: Categoría 1: Inocuo (< $30 \%)$; Categoría 2: Ligeramente toxico $(30-79 \%)$; Categoría 3: Moderadamente toxico (80-99\%); Categoría 4: Toxico (> 99\%).

\section{RESULTADOS Y DISCUSIÓN}

Compatibilidad de B. bassiana con larvas de $C$. externa

En la evaluación del hongo B. bassiana sobre el periodo del estadio I de C. externa, en condiciones controladas, se observaron diferencias significativas $(\mathrm{P}<0,05)$ entre las concentraciones evaluadas del entomopatógeno, en relación con el porcentaje de mortalidad del depredador, transcurridas 96 horas de exposición al hongo (Fig. 1).

Se observó que el tratamiento de $1 \times 10^{8}$ conidias $\mathrm{mL}^{-1}$ presentó la mayor mortalidad

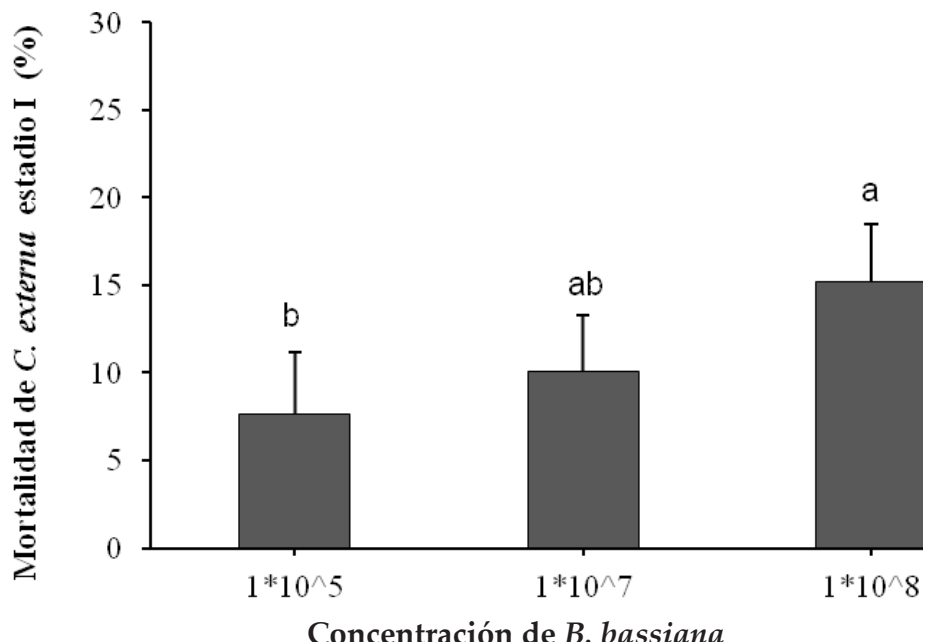

Fig. 1. Porcentajes de mortalidad corregida del primer estadio de $C$. externa expuesto a diferentes concentraciones de $B$. bassiana durante 96 horas en condiciones de laboratorio. Letras iguales indican que no se presentaron diferencias significativas, según la prueba de Tukey $(P \leq 0,05)$. Barras verticales representan error estándar.

Fig. 1. Corrected mortality rates of the first larval stage of $C$. externa exposed to different concentrations of $B$. bassiana for $\mathbf{9 6}$ hours under laboratory conditions. Same letters indicate no significant differences according to Tukey test $(\mathrm{P} \leq 0.05)$. Vertical bars represent standard error. 
$(15,2 \%)$, con diferencias significativas respecto de los tratamientos de $1 \times 10^{5}$ conidias $\mathrm{mL}^{-1}$ (7,61\%) y $1 \times 10^{7}$ conidias $\mathrm{mL}^{-1}(10 \%)$ (Fig. 1 ). La mortalidad en C. externa, por el uso de hongos entomopatógenos, se puede relacionar con dos características: momento de la aplicación y patogenicidad del hongo sobre el depredador (Vázquez et al., 2008). Ambos factores pudieron incidir en la mortalidad obtenida en el presente trabajo, en particular, el momento de aplicación, que correspondió a un estado inicial del desarrollo larval de C. externa (Estadio I) que por sus características morfológicas y fisiológicas tiende a ser más susceptible a cualquier tipo de agente nocivo externo.

En la evaluación sobre el estado larval de C. externa, estadio II y III, no se presentaron diferencias significativas entre las concentraciones evaluadas del entomopatógeno $(\mathrm{P}<0,05)$. Cuando se aplicó la prueba de Tukey, se estableció que no hubo grupos estadísticamente diferentes (Fig. 2 y 3), en relación con el porcentaje de mortalidad del depredador, transcurridas 96 y 192 horas de la exposición al hongo durante el periodo del estadio II y III respectivamente.

Según se observa en las figuras 2 y 3, no se presentó un efecto letal significativo sobre los estadios evaluados de C. externa; estos resultados concuerdan con los obtenidos por Pessoa et al.
(2005) y Estévez et al. (2011), quienes determinaron que no hubo efecto sobre la supervivencia del depredador en ninguna de las concentraciones que evaluaron $\left(1 \times 10^{7}\right.$ y $1 \times 10^{8}$ conidias $\left.\mathrm{mL}^{-1}\right)$ de la cepa IBCB6 de B. bassiana, obteniendo mortalidades por debajo del $20 \%$ en sus estudios. Por otro lado, Ceiro et al. (2011) reportaron valores de mortalidad de C. externa menores al 30\% con el hongo Pochonia chlamydosporia var. catenulata, confirmando la ausencia de patogenicidad de este microorganismo sobre el depredador.

Las anteriores evaluaciones demuestran la implementación y complementación que puede darse en el manejo integrado de plagas (MIP) con diversos métodos de control biológico. Dentro del MIP es importante también considerar el momento de la aplicación o implementación de los enemigos naturales, con base en el ciclo biológico de T. vaporariorum, y como relacionarlo con la liberación de depredadores, ya que autores como James et al. (2003) reportaron, que el segundo estadio de mosca blanca es más susceptible a $B$. bassiana que los otros estadios ninfales de este insecto plaga; otro factor a tener en cuenta es la influencia del microclima que se presenta en los invernaderos, especialmente la humedad relativa, debido a que se presenta un aumento durante el día, incrementando la transpiración del cultivo, lo que favorece la

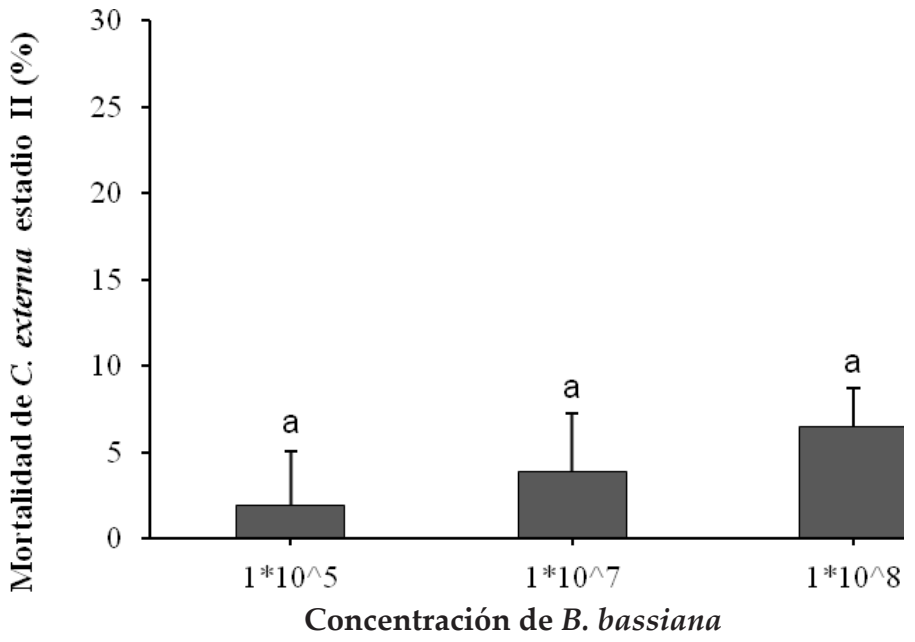

Fig. 2. Porcentaje de mortalidad corregida del segundo estadio de C. externa, expuestos a diferentes concentraciones de B. bassiana durante 96 horas en condiciones de laboratorio. Letras iguales indican que no se presentaron diferencias significativas, según la prueba de Tukey $(P \leq 0,05)$. Barras verticales representan error estándar.

Fig. 2. Corrected mortality rates of the second larval stage of $C$. externa exposed to different concentrations of B. bassiana for 96 hours under laboratory conditions. Same letters indicate no significant differences according to Tukey test $(P \leq 0.05)$. Vertical bars represent standard error. 


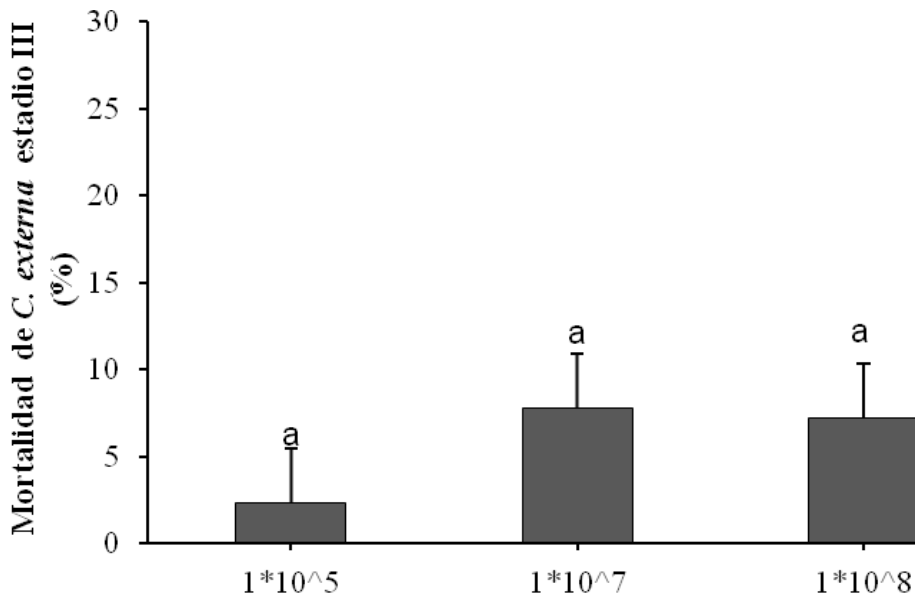

Concentración de B. bassiana

Fig. 3. Porcentaje de mortalidad corregida del tercer estadio de $C$. externa, expuestos a diferentes concentraciones de B. bassiana durante 192 horas en condiciones de laboratorio. Letras iguales indican que no se presentaron diferencias significativas, según la prueba de Tukey $(P \leq 0,05)$. Barras verticales representan error estándar.

Fig. 3. Corrected mortality rates of the third larval stage of $C$. externa exposed to different concentrations of B. bassiana for 192 hours under laboratory conditions. Same letters indicate no significant differences according to Tukey test $(P \leq 0.05)$. Vertical bars represent standard error.

acción de los hongos entomopatógenos (Espinel et al., 2008).

\section{Compatibilidad de M. anisopliae en larvas de $C$. externa}

En la evaluación del efecto del hongo $M$. anisopliae sobre el estado larval de C. externa, el porcentaje de mortalidad, no presentó diferencias significativas $(\mathrm{P}<0,05)$ entre las concentraciones evaluadas de $1 \times 10^{5}, 1 \times 10^{7}$ y $1 \times 10^{8}$ conidias $\mathrm{mL}^{-1}$ para los estadios analizados, trancurridas 96 horas para el primer y segundo estadio y de 192 horas para el tercer estadio de exposición al hongo.

En el primer estadio larval de C. externa (Fig. 4) se observó que M. anisopliae no afectó la supervivencia del depredador en ninguna de sus concentraciones, siendo la mortalidad similar en las tres concentraciones evaluadas, y oscilando entre 5 y $10 \%$; este valor fue semejante al obtenido en algunos estudios, como el realizado por Souza et al. (2015), quienes obtuvieron tan solo un 5\% de inviabilidad para el mismo depredador con este hongo. De acuerdo con Dacosta et al. (2008), un producto genera toxicidad cuando afecta la hemolinfa de la presa o de su controlador, proceso que no se presentó en el presente estudio, dado los rangos de mortalidad tan bajos que se obtuvieron.
Del mismo modo, individuos de C. externa en los estadios II y III no fue influenciada por el entomopatógeno $M$. anisopliae, puesto que se observaron mortalidades promedias de 5,8\% y $4,6 \%$, respectivamente (Fig. 5 y 6 ). Dichos valores concuerdan con los resultados de Schlick-Souza et al. (2011b), quienes reportaron que C. externa expuesta a concentraciones de $1 \times 10^{5}$ y $1 \times 10^{8}$ esporas $\mathrm{mL}^{-1}$ de $M$. anisopliae, no evidenció una mortalidad significativa en ninguno de sus estadios larvales, así como tampoco se afectó su capacidad depredadora sobre ninfas de Bemisia tabaci (Gennadius). Por su parte, Thunagrabeab y Tongma (2007) observaron una patogenicidad de tan solo el $4 \%$ con este mismo hongo entomopatógeno, sobre individuos de C. carnea, otra especie depredadora del género Chrysoperla, muy empleada en control biológico de plagas.

Las aplicaciones de M. anisopliae en general originaron mortalidades bajas en C. externa, inferiores al $10 \%$, lo anterior puede ser atribuible a la coevolución entre huéspedes y patógenos, argumento que explicaría las diferentes susceptibilidades que presentan las especies hacia sus enemigos naturales, y en particular en los hongos entomopatógenos que pueden ser muy específicos e infectar solo cierto tipo de hospederos (Thungrabeab y Tongma, 2007).

El periodo de infestación, germinación y 


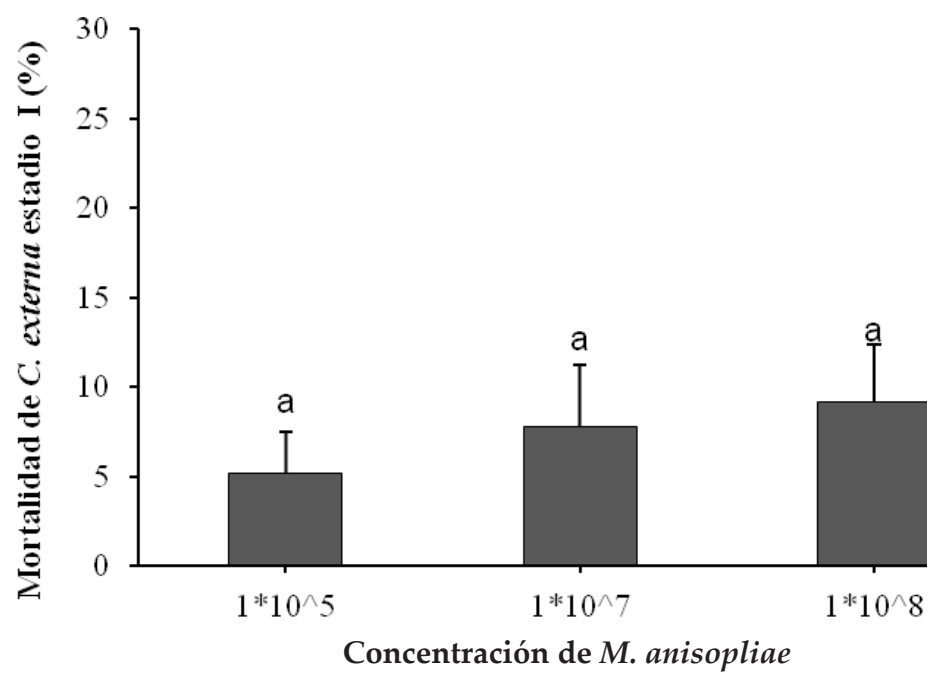

Fig. 4. Porcentaje de mortalidad corregida del primer estadio de $C$. externa, expuesto a diferentes concentraciones de $M$. anisopliae durante 96 horas en condiciones de laboratorio. Letras iguales indican que no se presentaron diferencias significativas, según la prueba de Tukey ( $P$ $\leq 0,05)$. Barras verticales representan error estándar.

Fig. 4. Corrected mortality rates of the first larval stage of C. externa exposed to different concentrations of M. anisopliae for $\mathbf{9 6}$ hours under laboratory conditions. Same letters indicate no significant differences according to Tukey test $(\mathrm{P} \leq 0.05)$. Vertical bars represent standard error.

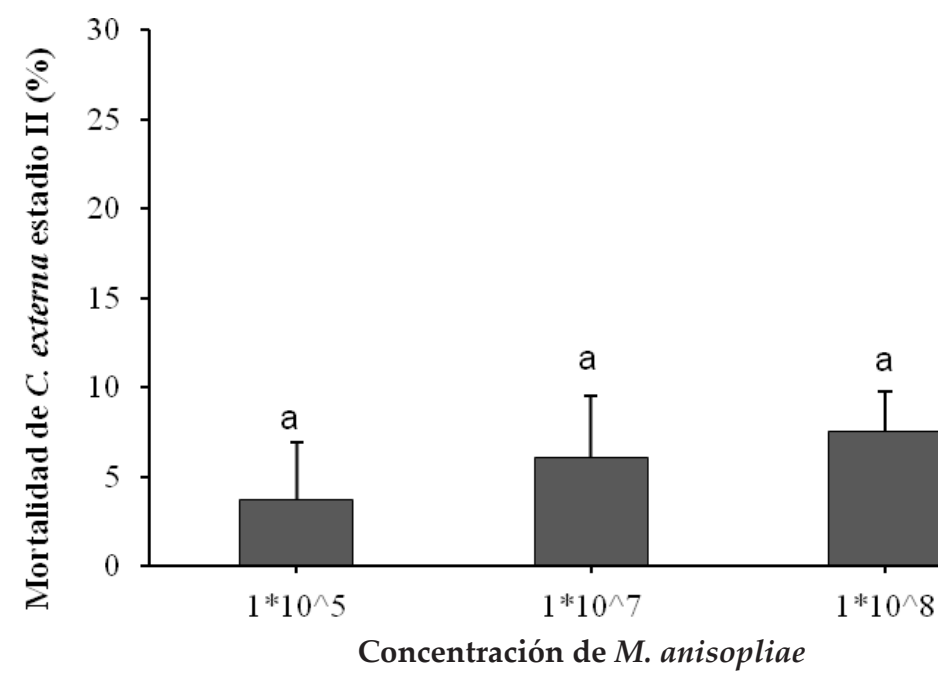

Fig. 5. Porcentaje de mortalidad corregida del segundo estadio de $C$. externa, expuestos a diferentes concentraciones de $M$. anisopliae durante 96 horas en condiciones de laboratorio. Letras iguales indican que no se presentaron diferencias significativas, según la prueba de Tukey ( $P$ $\leq 0,05)$. Barras verticales representan error estándar.

Fig. 5. Corrected mortality rates of the second larval stage of $C$. externa exposed to different concentrations of M. anisopliae for $\mathbf{9 6}$ hours under laboratory conditions. Same letters indicate no significant differences according to Tukey test $(\mathrm{P} \leq 0.05)$. Vertical bars represent standard error.

penetración de $M$. anisopliae, y B. bassiana se presenta en un lapso de tiempo de 18 horas, posteriormente, ocurre la colonización y esporulación del hongo después de entrar en contacto con su blanco biológico (Albuquerque y Albuquerque, 2009). Este proceso no se evidenció en los insectos que murieron, aún después de ocho días de observación en medio PDA; 


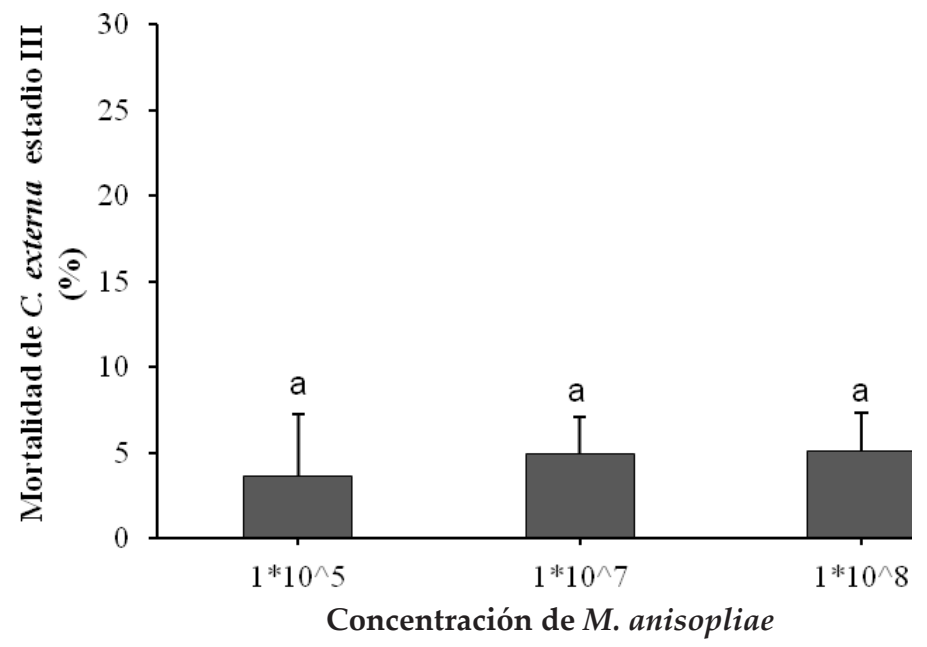

Fig. 6. Porcentaje de mortalidad corregida del tercer estadio de C. externa, expuestos a diferentes concentraciones de $M$. anisopliae durante 192 horas en condiciones de laboratorio. Letras iguales indican que no se presentaron diferencias significativas, según la prueba de Tukey ( $P$ $\leq 0,05)$. Barras verticales representan error estándar.

Fig. 6. Corrected mortality rates of the third larval stage of $C$. externa exposed to different concentrations of $M$. anisopliae for 192 hours under laboratory conditions. Same letters indicate no significant differences according to Tukey test $(P \leq 0.05)$. Vertical bars represent standard error.

adicionalmente, en las improntas realizadas en cada individuo no se verificó morfológicamente la presencia de los hongos evaluados. Lo anterior indica por la compatibilidad que se observó, que transcurridas 24 horas de las aplicaciones de los hongos B. bassiana y M. anisopliae, se puede realizar la liberación de C. externa.

Así mismo, en los individuos del depredador muertos y sometidos a cámara húmeda, no hubo desarrollo de micelio, lo cual puede ser debido a la no infección o a los diferentes mecanismos de defensa que presentan las larvas de la familia Chrysopidae a organismos que producen una patogénesis (Murata et al., 2006; Silva et al., 2006), dado que, en los insectos se desarrollan procesos como la melanización, fagocitosis, nodulacion y encapsulamiento (Hoffmann et al., 1999), que pueden inhibir el crecimiento de B. bassiana y $M$. anisopliae; razón por la cual se han considerado como hongos entomopatógenos relativamente seguros para enemigos naturales e insectos benéficos (Thungrabeab y Tongma, 2007).

En el estado larval se observó el adecuado desarrollo en cuanto a tamaño y movilidad, en los tres estadios del predador. Sin embargo, algunos autores consideran que los depredadores expuestos a entomopatógenos pueden presentar efectos subletales en su biología, comportamiento o ciclo de vida, que pueden llegar a afectar su capacidad de consumo y su progenie (Maia et al., 2000); no obstante, estos efectos no fueron cuantificados en el presente estudio, en virtud que otros autores como Amorim et al. (2005) y Souza et al. (2015) en las pruebas realizadas, descartan que se presenten algunos de estas consecuencias en el depredador C. externa.

De acuerdo con los resultados del presente estudio, aunque se observó algún nivel de mortalidad del estado larval de C. externa expuesta a concentraciones de $1 \times 10^{5}, 1 \times 10^{7}$ y $1 \times 10^{8}$ conidias $\mathrm{mL}^{-1}$ de B. bassiana y $M$. anisopliae, este no fue superior al $30 \%$, lo anterior permite clasificar a estos entomopatógenos como inocuos para el depredador evaluado, con base en la escala de toxicidad a enemigos naturales, aplicada por la OILB (Viñuela et al., 1993), Este resultado concuerda con lo obtenido por Estévez et al. (2011) en donde todos los estados de desarrollo fueron inocuos con porcentajes de mortalidad que oscilaron entre el $10 \mathrm{y}$ el $15 \%$ para el huevo, 12 y $20 \%$ para larva, 10 y $20 \%$ para pupa y $10 \%$ para el adulto, cuando fueron expuestos a $B$. bassiana cepa LBb-1. Lo anterior se puede deber a que $C$. externa presenta la capacidad de metabolizar o desintoxicarse cuando entra en contacto con estos entomopatógenos.

Los hallazgos en este trabajo y de otros autores, permiten visualizar una estrategia biológica integrada para el manejo de $T$. vaporariorum, en virtud que indican como viable, la combinación 
de múltiples agentes de control biológico que actúen sobre los diferentes estados de desarrollo del ciclo de vida de la mosca blanca (Islam et al., 2010; Sparfel, 2013).

\section{CONCLUSIONES}

Los resultados encontrados evidenciaron que los hongos entomopatógenos Beauveria bassiana (Bals.) Vuill. y Metarhizium anisopliae (Metsch.) bajo las condiciones evaluadas no tuvieron un efecto significativo en el segundo y tercer estadio larval de Chrysoperla externa Hagen.

El primer estadio larval de C. externa fue el más susceptible a la aplicación de B. bassiana, con un registro de mortalidad del $15 \%$ con la dosis de $1 \times 10^{8}$ conidias $\mathrm{mL}^{-1}$ del hongo.

La implementación conjunta de C. externa Hagen, B. bassiana y M. anisopliae, para el control biológico de Trialeurodes vaporariorum, puede llegar a ser una estrategia de manejo viable de acuerdo con la poca incompatibilidad observada.

Finalmente, es necesario realizar pruebas adicionales en condiciones de campo, para validar el uso de estos organismos de control biológico en el manejo de $T$. vaporariorum en el cultivo de tomate.

\section{RECONOCIMIENTOS}

A la Dirección de Investigaciones y Grupo Manejo Biológico de Cultivos de la Universidad Pedagógica y Tecnológica de Colombia por la financiación del proyecto SGI 1879.

\section{LITERATURA CITADA}

Abbott, W. S. 1925. A method of computing the effectiveness of an insecticidal. J. Econ. Entom. 18:265-267.

Albuquerque, E.A., y E.H. Albuquerque, 2009. Hongos entomopatógenos: importante herramienta para el control de "moscas blancas" (Homoptera: Aleyrodidae). An. Acad. Pernamb. Ciênc. Agron 5(6):209-242.

Agronet. 2018. Red de información y comunicación del sector agropecuario Colombiano, Ministerio de Agricultura. Disponible en http://www.agronet.gov.co/estadistica (Consulta: 21 de marzo de 2018).

Amorim, L., C. Sousa, A. Moino, y B. Souza. 2005. Compatibilidade entre Beauveria bassiana e o predador Chrysoperla externa em laboratório. Pesq. Agropec. Bras. 40(6):617-619.

Bai, Y., and P. Lindhout. 2007. Domestication and breeding of tomatoes: What have we gained and what can we gain in the future? Ann. Bot. 100:1085-1094.
Barrera, J., J. Carrascal, S. Numa, D. Rodríguez, y F. Cantor. 2013. Compatibilidad de Encarsia formosa (Hymenoptera: Aphelinidae) con productos comerciales en condiciones de laboratorio. Acta Biol. Colomb. 18(2):265-270.

Bautista, L., J. Cardona, A. Soto, y P. Vélez. 2014. Actividad entomopatógena de tres hongos sobre Hortensia similis (Hemiptera: Cicadellidae) y Collaria scenica (Hemiptera: Miridae) en sistemas silvopastoriles. Bol. Cient. Mus. Hist. Nat. 18:188-196.

Barnett, H.L., and B.B. Hunter. 1998. Ilustrated genera of imperfect fungi. 240 American Phytopathology Society, Saint Paul, Minnesota, USA.

Cardona, C., F. Rendón, J. García, A. López, J.M. Bueno. J.D., y Ramírez. 2001. Resistencia a insecticidas en Bemisia tabaci y Trialeurodes vaporariorum (Homoptera: Aleyrodidae) en Colombia. Rev. Col. Entomol. 27(1-2):33-38.

Castro, M., J. Martínez, y M. Dotor. 2016. Evaluación del efecto regulador de Chrysoperla externa sobre mosca blanca Trialeurodes vaporariorum en tomate. Rev. Cienc. Agr. 33(2):4354.

Ceiro, W., A. Puertas, J. Arévalo, y L. Hidalgo-Díaz. 2011. Efectos de la aplicación de Pochonia chlamydosporia var. catenulata Kamyscho ex Barron y Onions (Zare y Gams) sobre el desarrollo de plántulas de tomate (Solanum lycopersicum L.). Rev. Protección Veg. 26(2):118-121.

Dacosta, R.R., G.A. Carvalho, C.F. Carvalho, e R.R. Costa. 2008. Ação de fungicidas utilizados na cultura do pepino sobre larvas de primeiro ínstar de Chrysoperla externa (Hagen, 1861) (Neuroptera: Chrysopidae) e os efeitos sobre suas fases subseqüentes. Arq. Instit. Biol. 75(3):343-350.

Espinel, C., M. Lozano, L. Villamizar, E. Grijalba, y A. Cotes. 2008. Estrategia MIP para el control de Bemisia tabaci (Hemiptera: Aleyrodidae) en melón y tomate. Rev. Col. Entomol. 34(2):163-168.

Estévez, O., E. Massó, R. Abreu, y D. Baró. 2011. Susceptibilidad de Chrysopa exterior Navás a Beauveria bassiana (Balsamo) Vuillemin cepa lbb-1 en condiciones de laboratorio. Fitosanidad 15(1):51-57.

Gamboa, S., B. Souza, y R. Morales. 2016. Actividad depredadora de Chrysoperla externa (Neuroptera: Chrysopidae) sobre Macrosiphum euphorbiae (Hemiptera: Aphididae) en cultivo de Rosa sp. Rev. Col. Entomol. 42(1):54-58.

Hajek, A.E. 1997. Ecology of terrestrial fungal entomopathogens. Adv. Microb. Ecol. 15:193249. 
Hamdi, F., J. Fargues, G. Ridray, B. Jeannequin, and O. Bonato. 2011. Compatibility among entomopathogenic hipocreales and two beneficial insects to control Trialeurodes vaporariorum (Hemiptera: Aleyrodidae) in mediterranean greenhouses. J. Invertebr. Pathol. 108: 22-29.

Herrera, J., A. Hurtado-Salazar, y N. Ceballos-Aguirre. 2015. Estudio técnico y económico del tomate tipo cereza élite (Solanum lycopersicum L. var. cerasiforme) bajo condiciones semicontroladas. Rev. Col. Cienc. Hort. 9(2):290-300.

Hoffmann, J., F. Kafatos, C. Janeway, and R. Ezekowitz 1999. Phylogenetic perspectives in innate immunity. Science 284(5418):1313-1318.

IOBC/WPRS. 1992. Working Group "Pesticides and Beneficial Organisms". Guidelines for testing the effects of pesticides on beneficial organisms: description of test methods. International Organization for Biological and Control of noxious animals and plants (IOBC)/ West Palearctic Regional Section (WPRS). Bulletin IOBC/WPRS 15:1-186.

Islam, M. T., S. J. Castle, S. Ren. 2010. Compatibility of the insect pathogenic fungus Beauveria bassiana with neem against sweetpotato whitefly, Bemisia tabaci, on eggplant. Entomol. Exp. Appl. 134:28-34.

James, R. R., J. S. Buckner, and T. P. Freeman. 2003. Cuticular lipids and silver leaf whitefly stage affect conidial germination of Beauveria bassiana and Paecilomyces fumosorose us. J. Invertebr. Pathol. 84(2):67-74.

Jaramillo, J., V. Rodríguez, M. Guzmán, M. Zapata, y T. Rengifo. 2007. Manual técnico: Buenas prácticas agrícolas en la producción de tomate bajo condiciones protegidas. $331 \mathrm{p}$. FAO - Gobernación de Antioquia, Corpoica, Centro de Investigación "La Selva", Rionegro, Colombia.

Lucero, A., L. Peña, y T. Bacca. 2004. Evaluación de la actividad biocontroladora de Beauveria bassiana y Metarhizium anisopliae sobre larvas de Ancognatha scarabaeiodes (Coleoptera: Scarabaeidae). Revista Corpoica: Cienc. Tecnol. Agropecuaria 5(1):43 - 48.

Maia, W., C. Carvalho, e B. Souza. 2000. Exigências térmicas de Chrysoperla externa (Hagen, 1861) (Neuroptera: Chrysopidae) alimentada com Schizaphis graminum (Rondani, 1852) (Hemiptera: Aphididae) em condições de laboratório. Ciênc. Agrotec. 24:81-8.

Martínez, O., E. Ebratt, W. Turizo, G. Guerrero, and R. Acosta. 2012. Presence of Bemisia tabaci (Hemiptera: Aleyrodidae) and Begomovirus, associated with tomato crops Solanum lycopersicum L. in Cundinamarca. Agron. Colomb. 30(3):395-402.
Monzón, A. 2001. Producción, uso y control de calidad de hongos entomopatógenos en Nicaragua. Manejo Integrado de Plagas 63:95-103.

Murata, A.T., A.C. Caetano, S.A. Bortoli, y C.H. Brito. 2006. Capacidad de consumo de Chrysoperla externa (Hagen, 1861) (Neuroptera: Chrysopidae) em diferentes presas. Rev. Caatinga 19:304-309.

Perrilla, A., L. Rodríguez, y L. Bermúdez. 2011. Estudio técnico-económico del sistema de producción de tomate bajo invernadero en Guateque, Sutatenza y Tenza (Boyacá). Rev. Col. Cienc. Hort. 5(2):220-232.

Pessoa, L., G. Cavalcanti, R. Sousa, M. Alcides, e B. Souza. 2005. Compatibilidade entre Beauveria bassiana e o predador Chrysoperla externa em laboratório. Pesqui. Agropecu. Bras. 40(6):617-619.

Schlick-Souza, E., L. Toscano, G. Souza-Schlick, W. Maruyama, e A. Andrade. 2011a. Desenvolvimiento larval de Chrysoperla externa alimentada con Aphis gossypii provenientes de três cultivares de algodoeiro. Revista Agrarian Dourados 4(13):182-188.

Schlick-Souza, E.C., L.C. Toscano, G.D. Souza-Schlick, E. Adriano, W.I. Maruyama, e A.J. Peres. 2011b. Capacidade predatória de Chrysoperla externa sobre Bemisia tabaci biótipo B expostas ao fungo Metarhizium anisopliae. Sci. Agrar. 12(2):121-126.

Silva, A.R., P.R. Reis, B. Souza, C.F. Carvalho, G.A. Carvalho, V.L. Cosme. 2006. Flutuação populacional de adultos de Chrysoperla externa (Hagen, 1861) (Neuroptera: Chrysopidae) em cafeeiros conduzidos em sistemas orgânico e convencional. Manejo Integrado de Plagas 77:44-49.

Souza, E., L. Toscano, G. Schlick, A. Peres, P. Dias, e W. Maryama. 2015. Compatibilidade de Metarhizium anisopliae (Metschnikoff) Sorokin (Hypocreales: Clavicipitaceae) com Chrysoperla externa (Hagen) (Neuroptera: Chrysopidae). Entomo Brasilis, 8(3):189-195.

Sparfel, M. 2013. Compatibility of entomopathogens in controlling whitefly Bemisia tabaci. 18 p. University of Florida, Florida, USA.

Téllez, A., M. Cruz, Y. Mercado, A. Asaff, y A. Arana. 2009. Mecanismos de acción y respuesta en la relación de hongos entomopatógenos e insectos. Rev. Mex. Mic. 30(1):73-80.

Thungrabeab, M. and S. Tongma. 2007. Effect of entomopathogenic fungi, Beauveria bassiana (Balsam) and Metarhizium anisopliae (Metsch) on non-target insects. KMITL Sci. Tech. J. 7:812. 
Vázquez, L., C. Murguido, A. Elizondo, O. Elósegui, y F. Morales. 2007. Control biológico de la mosca blanca Bemisia tabaci. Instituto de Investigaciones de Sanidad Vegetal (INISAV). Publicación CIAT 355:1-56.

Vélez, P., F. Posada, P. Marín, M. González, E. Osorio, y A. Bustillo. 1997. Técnicas para el control de calidad de formulaciones de hongos entomopatógenos. 37 p. Boletín Técnico No. 17, Cenicafé, Chinchiná, Caldas, Colombia.

Viñuela, E.; J.A. Jacas; V. Marco; A. Adan; y F. Budia. 1993. Los efectos de los plaguicidas sobre los organismos beneficiosos en agricultura. Grupo de trabajo de OILB Plaguicidas y Organismos Beneficiosos I. Insecticidas y acaricidas. Phytoma 45:18-25. 